\title{
Neurapraxia in an infected radicular cyst and its management
}

\author{
Abhinav Kumar 1,*, Parag N. Kisave ${ }^{2}$, Nandeibam Premita ${ }^{3}$, Preetam Kumar Gupta ${ }^{4}$, Prachi $^{5}$ \\ ${ }^{1,2,3} \mathrm{PG}$ Trainee, ${ }^{4} \mathrm{BDS},{ }^{5}$ Tutor, ${ }^{1,2}$ Dept. of Oral and Maxillofacial Surgery, ${ }^{3}$ Dept. of Oral Medicine and Radiology, ${ }^{4,5} \mathrm{Dept}$. of \\ Dental Surgery, Vyas Dental College \& Hospital, Jodhpur, Rajasthan, India
}

\section{*Corresponding Author: Abhinav Kumar}

Email: abhinavbabbu.singh@gmail.com

\begin{abstract}
Radicular cysts are the most common inflammatory odontogenic cyst of jaw that emanates from the epithelial cell rests of malassez in the periodontal ligament. $60 \%$ of cases are found in maxilla, most commonly around incisors and canines with male predilection. Most of the cases remain silent until secondarily infected. Treatment modalities of Radicular cyst according to their size and severity are extraction of the associated tooth, endodontic treatment, apical surgery, enucleation and/or marsupialization. Though, radicular cyst involving inferior alveolar nerve may cause paraesthesia due the neurosensory disturbance caused by mechanical compression or stress. Here, we report a case of neurapraxia occurred in an infected radicular cyst involving roof of the inferior alveolar nerve canal.
\end{abstract}

Keywords: Radicular cyst, Inferior alveolar nerve, Paraesthesia, Neurosensory disturbance, Compression, Stress.

\section{Introduction}

Eponymous terms of radicular cyst are periapical cyst, apical periodontal cyst or root end cyst or dental cyst. The main causative agent is trauma or infected tooth. They occur in between $52-68 \%$ in human jaws. ${ }^{1}$ Most commonly occur in Maxilla and around incisors and canines. ${ }^{2}$ Small cyst of radicular cyst can often heal by endodontic treatment without any surgical procedure though larger one need surgical procedure. ${ }^{3}$ Treatment is depend on the size, location of the lesion, integrity of the bone, wall of the cystic, their presence near the vital structures. ${ }^{4}$ However, larger radicular cyst may expand through the associated innervated nerves. Inferior alveolar nerve paraesthesia associated with periapical pathosis is usually related to compression induced by an acute inflammatory response such as edema or abscess formation. ${ }^{5,6}$

According to Seddon's classification, peripheral neuropathies classified into three types. ${ }^{7}$ Neuropraxia (mild form of injury); in this type of injury, there is little or no structural damage with no loss of nerve continuity. Their symptoms are short in duration and belief that they result from a mixture of mechanical compression and ischaemia. Their recovery is reversible, unless ischaemia lasts roughly 8 hours. Example of this type of injury is carpal tunnel syndrome and it is caused by the pressure on the arm after the person has fallen asleep, usually during an alcoholic binge. It is normally recovered within weeks or months. ${ }^{8}$ Axonotmesis is the type of injury in which there is complete interruption of the nerve axon and its myelin sheath, but the mesenchymal structures including perineurium and epineurium are either completely or partially intact. Example is laceration caused by the broken glass. Recovery of this type of injury depend on extend of the injury, with increasing severity related to poorer outcome. ${ }^{8}$ Though according to Morgan GW. (2003), their recovery can take can take
3 to 4 months if there is more severe damage to the nerve. ${ }^{9}$ Neurotmesis occurs when a nerve, along with its surrounding stroma, becomes completely disconnected. There is no spontaneous recovery and even after surgery prognosis is poor. The only example of this type of injury is seen in major trauma. ${ }^{8}$ Here, we are reporting a case of Neurapraxia initiated by mechanical stress or compress during the extraction.

\section{Case Report}

A 46 years old male patient came to department of Oral Medicine and Radiology Department with the chief complaint of swelling in the right lower front of jaw since 10-15 days (Fig. 1). Patient revealed history of root stump since 2years and pimple like swelling since 1 year and drained the abscess. There was no history of pain and bleeding though he felt pain since 6 months back. There was no contributory history of past medical, dental, familial and allergic drugs. Extraorally, there was no facial asymmetry and mouth opening was normal. Intraorally, a diffuse solitary swelling was present from mesial aspect of 42 till distal aspect of canine. It was soft in consistency and non-tender. The shape was dome-shaped and size is roughly $(2 * 2) \mathrm{cm}$ in dimension. Root stump present in relation to 45 , grossly decayed tooth irt 38 and missing teeth irt. 16. So, according to history and clinical manifestations, we gave the diagnosis Infected Radicular Cyst irt. 45.

We advised OPG so that we can find out the radiographic features of the lesion and to rule out the extension of the lesion and to see the resorption or displacement of the teeth. 


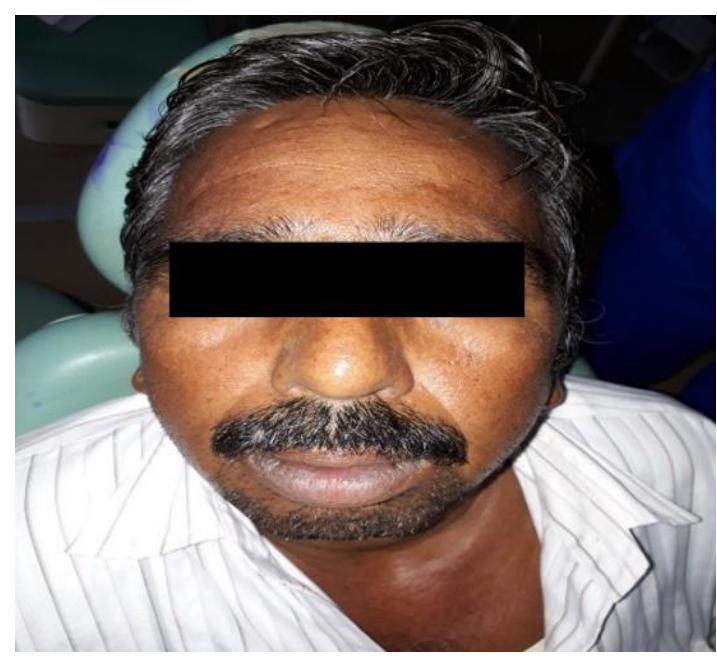

Fig. 1: Extraoral profile

Histologically, one bit of tissue showing nonkeratinized stratified squamous epithelium with varying arcading rete-ridges covering a richly cellular connective tissue capsule, connective tissue is exhibiting dense infiltration of chronic inflammatory infiltrated, varying sized vascular spaces with extravasated RBCs can also be noted on E\&H stained section.

According to the radiographic findings (Fig. 2), root stump was present in relation to 45 and periapical abscess in relation to 38. Well defined unilocular radiolucency was seen and antero-posteriorly extending from distal aspect of 44 root till mesial aspect of 46 root. The size was roughly $(2 \times 2.5) \mathrm{cm}$ in dimension.Supero-inferiorly extending from apical third of the roots till roof of mental nerve. The posterior aspect was surrounded by corticated border and external root resorption is seen in relation to 44,45 and mesial root of 46 . We found out that there is possiblity of injuring the mandibular nerve as we need to compress or stretch the mental nerve during the surgical procedure and more chances of causing paraesthesia. So, we informed the patient about consequences of the procedure and took consent also.

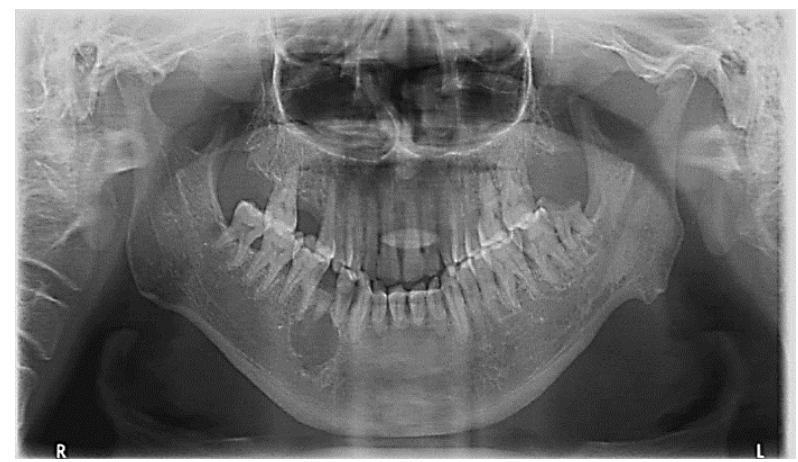

Fig. 2
Root stump is present in relation to 45 and periapical abscess in relation to 38 . Well defined unilocular radiolucency is seen from distal aspect of 44 root till mesial aspect of 46 root. The size is roughly $(2 \times 2.5) \mathrm{cm}$ in dimension and is involved inferior alveolar nerve canal roof. The posterior aspect is surrounded by corticated border and external root resorption is seen in relation to 44,45 and mesial root of 46.

Required nerve block were given (inferior alveolar nerve block, long buccal,mental, lingual nerve block and local infiltration).We had done extration of 45 . Mesial releasing Incision was made from mesial aspect of canine (43) and distal releasing incision from distal aspect of first molar (46). Further crevicular incision was made using BP handle no. 3 and blade no. 15 from 43 till 46. By using periosteal elevator and Howarth elevator, the flap was raised. Defective part of bone was excised by using Allis forcep as it help in holding the cystic part and its lining. The exposed bone surface was covered by granulation tissue and it became smooth after cystic enucleation (Fig. 3\&4). Currettage was done in the herniated cystic bony cavity by he help of currette. Peripheral Osteotomy done, saline and betadine ( $2 \%$ povidione) irrigation done. Continuous sling suture given with 3.0 silk so that papilla and incision lines are brought as close as possible. We recalled the patient after 7 days though he gave history of numbness on right lip and chin that persists more than the local anaesthetic working hours i.e, 10 hours after surgery (it indicates involvement of mental nerve and inferior alveolar nerve).On second day, area of paraesthesia is recorded and diagnosed Neorosensory disturbances (Fig. 5). We prescribed neurobion forte bid for 30 days. On sixth-seventh day, there was improvement of NSD and he felt pain, which indicates recovery. On tenth day, sutured was removed and healing was appropriate (Fig. 6). Further, we adviced for intentional root canal treatment irt. 44 and 46. 

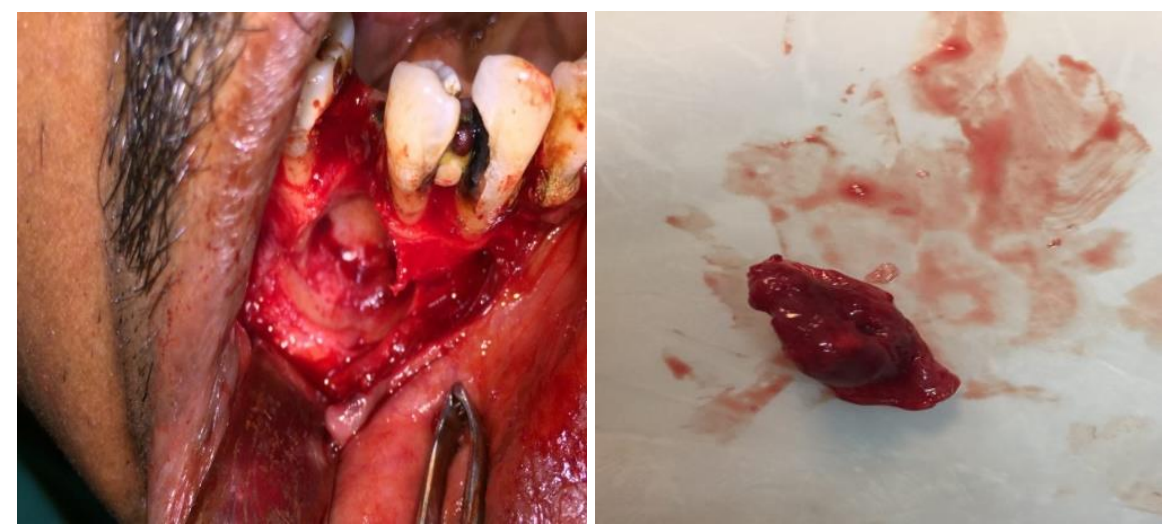

Fig. 3\&4: Defect bone seen after enucleation of the cystic lesion. The involved bone surface was resorbed and there was presence of scalloped bone surface; Enucleated lesion
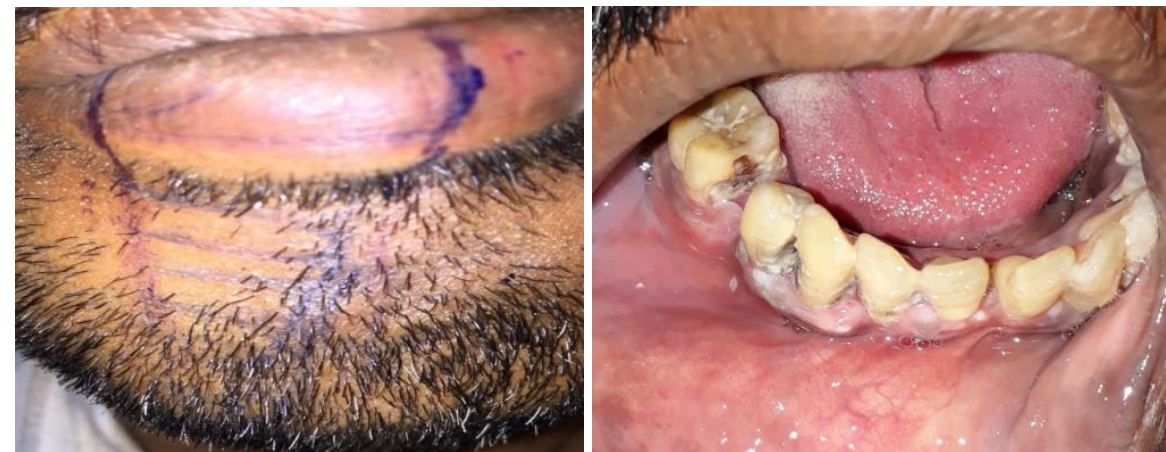

Fig. 5\&6: Mapping done on the involved surface; right lower lip and medial portion of chin; the affected area was entirely healed (post treatment picture).

\section{Discussion}

Radicular cyst can be defined as "if a well-defined radiolucency is observed at the apex of an untreated asymptomatic tooth with a non-vital or diseased pulp, and if the anatomic structures can be ruled out, the radiolucency is a dental granuloma or a radicular cyst in approximately $90 \%$ of the cases" (According to Wood and Goaz). ${ }^{10}$ They are occur in all tooth-bearing areas of the jaws. Prevalence rate is $60 \%$ in the maxilla especially maxillary anterior region and $40 \%$ in the mandible. Occurrence of radicular cyst in deciduous teeth is very rare. ${ }^{11}$

Nerve injuries can be result from compression, laceration or traction and their recovery is depend on the severity it is concussion type of injury. Their recovery starts within few days and complete in a few weeks. ${ }^{12}$ Nerve compression can occur in chronic case of radicular cyst due to increase in size that cause resorption of bone. ${ }^{1}$ According to Suhail L. et al (2009), two theories regarding the formation of radicular cyst cavity are suggested; i) The nutritional deficiency theory (they assume that there is nutritional deficiency in the central cells of epithelial strands and undergoes necrosis and liquefactive degeneration to form the cyst cavity lined by stratified epithelium). ii) Abscess theory (in this theory they suggest proliferating epithelium lines an abscess cavity formed by tissue necrosis and

lysis because of the innate nature of the epithelial cells to exposed connective tissue surfaces). ${ }^{13}$

The lesions which can cause Inferior alveolar nerve paraesthesia due to the simple mechanical compression to the inferior alveolar neurovascular bundle are odontoma, intraosseous lipogranuloma, traumatic bone cyst, or dentigerous cyst. ${ }^{14}$

How to diagnose the peripheral neuropathies? According to Caissie R. et al. (2005), the clinical assessment should consist of the given elements; ${ }^{15}$

Map the affected area by pencil outline on a drawing or a photograph of the patient.

1. Determine the sensations felt by the patient when a cotton swab is lightly applied to the affected area.

2. Determine the ability of the patient to detect the direction of a sweeping motion (in an area of about $1 \mathrm{~cm}$ ), applied with a resin applicator or the tip of a rolled-up tissue.

3. Describe the patient's sensations when a 27-gauge hard needle is applied in the affected region with sufficient pressure to indent the skin without penetrating it.

Dysesthesia or spontaneous pain presence must be noted. Each test must be conducted at 3 sites: the lower lip, the lip-chin fold and the chin. Patient with paresthesia should take corticosteroids after the surgery to decrease the inflammatory response (usually prednisone 50mg per day for 7 days). ${ }^{16}$ 
Simultaneously, patient should start antibiotic penicillin for 7 days and clindamycin for those who are allergic to penicillin. Clinical assessment should be done once in a month to rule out the functional recovery. ${ }^{15}$

In this case we encountered neurosensory disturbance i.e., neurapraxia which was ellevated by the mechanical stress and compression of involved nerve during the surgical procedure. We diagnosed the case in time and had given the necessary treatment. It was subsided completely after 10 days and the patient was satisfied with our treatment. We informed him to visit every month for 1 year duration to see any recurrence.

\section{Conclusion}

Diagnosis of neurosensory disturbance is very shcrucial to prevent permanent damage of the involved nerve. So, we need to be careful while assessing the patient and should diagnose as early as possible. Necessary precaution must be taken during the surgical procedure and the clinician should have sound knowlegde of the possible consequences so that can inform the patient before the treatment. In our case, we diagnosed the case as early as possible and treated in time without any delay.

\section{References}

1. Agarwal P, Sharma S, Trehan M, Vashistha A. Management of Infected Radicular Cyst by Marsupialization. World Journal of Dentistry. 2013;4(3):214-16.

2. White S.C, Pharoah M.J. Oral Radiology Principles and interpretation. $6^{\text {th }}$ Edition 2009;343p.

3. Nair PNR. Review new perspectives on radicular cysts: do they heal? International Endodontic Journal. 1998;31:155-60.

4. Kumar JA, Achuthan N, Loganathan K, Augustine D. Effective Management of a Large Radicular Cyst with Surgical Enucleation. Oral and Maxillofacial Pathology Journal. 2014;5(1):459-61.
5. Giuliani M, Lajolo C, Deli G, Silveri C. Inferior alveolar nerve paraesthesia caused by endodontic pathosis: a case report and review of the literature. Oral Surgery Oral Medicine Oral Pathology Oral Radiology Endodontics. 2001;92:670-4.

6. Morse DR. Infection-related mental and inferior alveolar nerve paresthesia: literature review and presentation of two cases. Journal of Endodontics. 1996;7:457-60.

7. Seddon HJ. Three types of nerve injury. Brain (a journal of neurology) 1943;66:247-88.

8. Burnett MG, Zager EL. Pathophysiology of peripheral nerve injury: a brief review. Neurosurgery Focus 2004 Article 1, 16 (5).

9. Morgan GW. Proprioception, touch and vibratory sensation. In: Goetz CG, Pappert EJ, eds. Textbook of clinical neurology, 2nd ed. Philadelphia: WB Saunders; 2003:333-50.

10. Wood NK, Goaz PW. Differential diagnosis of oral lesions, 5th ed. Philadelphia: Mosby; 1997:41.

11. Shear M and Speight P.M. Cyst of oral and maxillofacial region; $4^{\text {th }}$ edition 2006.

12. Sooriakumaran P, Jayasena C, Scully C. Key Topics in Human Diseases for Dental Students. 2005;177-81.

13. Suhail L, Ajaz AS, Suhail MJ. Radicular cyst. JK Science. 2009;11(4):187-189.

14. Hamada Y, Yamada H, Hamada A, Kondoh T, Suzuki M, Noguchi K, Ito K, Seto K. Simultaneous Paresthesia of the Lingual Nerve and Inferior Alveolar Nerve Caused by a Radicular Cyst. Journal of Endodontics. 2005;31(10):764-66.

15. Caissie R, Goulet J, Fortin M, Morielli D.Iatrogenic Paresthesia in the Third Division of the Trigeminal Nerve:12 Years of Clinical Experience. Journal of Canadian dental association. 2005;71(3):185-90.

16. Gregg JM. Nonsurgical management of traumatic trigeminal neuralgias and sensory neuropathies. Oral Maxillofacoal Clinical North America. 1992;4(2):375-92. 